\title{
Téoros
}

Revue de recherche en tourisme

\section{La méthode Delphi en question : tourisme en Suisse en l'an 2010}

\section{Jorg Hartwig}

Volume 12, numéro 2, juillet 1993

La prévision-prospective du tourisme

URI : https://id.erudit.org/iderudit/1078021ar

DOI : https://doi.org/10.7202/1078021ar

Aller au sommaire du numéro

Éditeur(s)

Université du Québec à Montréal

ISSN

0712-8657 (imprimé)

1923-2705 (numérique)

Découvrir la revue

Citer cet article

Hartwig, J. (1993). La méthode Delphi en question : tourisme en Suisse en l'an 2010. Téoros, 12(2), 18-21. https://doi.org/10.7202/1078021ar d'utilisation que vous pouvez consulter en ligne.

https://apropos.erudit.org/fr/usagers/politique-dutilisation/ 


\title{
La méthode Delphi en question : tourisme en Suisse en I'an 2010
}

\author{
Jorg Hartwig*
}

La prospective Delphi portant surl'avenir du tourismeen Suisse, menée en 1991 par trois anciens disciples du professeur docteur Jost Krippendorf ${ }^{11}$ ) n'a pas le caractère audacieux et novateur distinguant la même étude conduite par leur maître il y a quinze ans. La lecture de l'enquête $1991^{(0)}$ s'avère néanmoins fortement intéressante, et ce pour plusicurs raisons: non seulement constitue-t-elle une nouvelle source d'inspiration et de base de réflexion pour les intervenants du tourisme suisse mais elle comporteégalementune comparaisond'un certain nombre de pronostics de 1977 avec les avis des experts en 1991. En outre, certains pronostics de 1977 sont confrontés à la réalité des chiffres actuels, ce qui permetunepremièreévaluation dela aforce deprédictions du Delphi 1977, voire de la méthode elle-même.

\section{Delphi 1991}

Qualifiépar ses auteurs de *sortedecadeau d'anniversaires à l'occasion des 50 ans de fondation des deux instituts de recherches en tourisme à l'Université de Bern et St. Gallen ${ }^{(3)}$, HansruediMüller, Claude Kaspar et Hanspeter Schmidhauser ont organisé leur enquête en respectant largement méthode, contenu, horizon temporel et choix des personnes interrogées du Delphi 1977:

La prospective Delphi a de nouveau été retenue car les méthodes classiques de pronostic quantitatives sont difficilement à mettre en oeuvre dans le domaine du tourisme qui connaît des ruptures de tendances frequentes ${ }^{(4)}$. Müller, Kaspar et Schmidhauser se contentent cependant d'un Delphi a 2 tours au lieu de 3 tours en 1977. L'expérience de la première étude justifiait en effet cette aconcentrations, le troisième tour n'apportant quasiment pas d'informations supplémentaires.

Monsieur Jorg Hartwig est chercheur à l'Institut de recherche et didtudes superieures du Tourisme. Université de Paris I. Panthéon Sorbonne.
Les sujets abordés par l'étude sont les mêmes qu'en 1977; l'évolution dela demande touristique, de l'hébergement et du transport, la rentabilitédu secteur touristique et la disposition à l'investissement $y$ figurent au même titre que l'intervention des pouvoirs publics ainsi queles forces et faiblesses du tourisme en Suisse, et ce à l'horizon de l'an 2010. L'étude 1991 est cependant moins complète que celle menée par Krippendorf qui comportait en plus des questions sur l'aspect de la politique régionale et sur le marketing des entreprises touristiques.

Cinquante-deux experts de toutes les branches du tourisme ont été interrogés entre novembre 1990 et juin $1991 ; 17$ d'entre euxavaient déjà participéau premier Delphi 1977.

Le rapport présente leurs réponses une a une, verbalement et en chiffres (moyenne arithmétique des avis des experts après le deuxième tour du questionnaire et quartiles). On y trouve également une représentation graphique des résultats, un résumé des raisons intéressantes motivant chaque réponse, ainsi qu'un commentaire bref.

Nous avons acondensé les résultats chiffrés (cf, tableau 1) et citons nombre d'idées motivant les réponses (cf. encart E2).

\section{Comparaison : Delphi 1991 . Delphi 1977}

Delphi 1991 reprend la plupart des questions qui avaient été posées en 1977 . Cependant, parmi le nombre de pronostics, il n'y en a que sept qui sont véritablement comparables aux avis des experts en 1977 (lesquestions portantsur l'évaluation d'une diminution ou d'une croissance, plus ou moins forte, d'un phénomène n'ont pas les mêmes bases de départ en 1977 et 1991 et sont par conséquent difficilement comparables).

Deux des sept questions provoquent les mémes résultats en 1977 et en 1991: les touristes du Japon, États-Unis, Canada, Australie et Amérique du Sud ne constitueraient jamais $50 \%$ de l'activité touristique en Suisse et une obligation légale d'utilisation des résidences secondaires n'y existerait jamais (ou au moins pas avant 2010).

Ils'agit là depronosticssans aucune surprise avec une forte probabilité.

Ence qui concerne les cinq autres questions qui permettent une comparaison effective des réponses, les experts ont fortement changé d'avis depuis 1977: tandis que Delphi 1977 annonçait 70 millions de nuitées de la *parahôtellerie en Suisse en l'an 2010 (34 millions en 1975), le même pronostic des expertsen 1991 se monteà 40 millions de nuitées seulement ( 40 millions en 1989).

Cette «adaptation $*$ du pronostic selon une évolution récente met en question une des particularités de la méthode Delphi qui devait éviter les simples extrapolations et étre en partie fondée sur l'intuition des experts.

La question concernant l'évolution de la répartition des nuitées été/hiver démontre une autre faiblesse de la méthode: les experts avaient bien prévu la tendance et leur pronostic est une réalité à l'heure actuelle; par contre ils n'avaient pas prévu linversion de la tendance annoncée par Delphi 1991. Cet exemple nous montre quedanslecasd'une évolution non-linéaire, les fluctuations et changements de tendance qui se produisent entre formulation et échéance du pronostic ne sont pas pris en compte par la méthode Delphi.

\section{"Force de prédiction" du Delphi 1977}

Très peu de réponses de 1977 peuvent à I'heure actuelle être jugées définitivement sur leur concordance avec la réalité. Il y a des réponses qui semblent vraisemblables ou invraisemblables aujourd'hui mais 
l'avenir d'ici l'an 2010 nous réservera sûrement des surprises!

Quelques résultats peuvent cependant d'ores et déjà être classés dans les «faux pronosticss comme par exemple la croissance des remontées mécaniques (la réalité a dépassé les chiffres annoncés depuis longtemps), ou celui des touristes internationaux (Delphi 1977 avait annoncé un doublement des arrivées de touristes internationaux pour l'an 2000 correspondant à 440 millions de touristes. Déjà en 1992, ce chiffre était de 476 millions selon l'Office mondial du tourisme).

D'autres pronostics semblent peu probables comme par exemple la capacité de la «parahôtelleries que les experts avaient prévue pour l'an 2010 (cf. tableau 1).

D'autres prévisions de 1977 encore correspondent aux attentes des experts en 1991 et ont de fortes chances de se réaliser à l'échéance 2010.

Toutes ces observations mettent en évidence un manque certain de fiabilité de la méthode.

Après ces constats, peut-on toujours croire en la «force de prédictions du Delphi? Peut-on croire en l'utilité d'une méthode qui se veut être un outil permettant d'anticiper des ruptures de tendances tout en se basant sur une moyenne statistique qui lisse les réponses extrêmes ou surprenantes? D'autres critiques pourraient être faites mais la méthode garde certainement sa raison d'être si on la considère comme simple base de réflexion. Il faudrait par contre se méfier d'en faire un outil de décision et de gestion opératoire.

Ceci dit, n'est-il pas plus judicieux de *construire l'avenir»? *Construires en partant de son idéal et en essayant de faire en sorte que l'avenir y ressemble au lieu de s'adapter à une tendance pronostiquée. Faut-il se faire à la fatalité de l'oracle? Pourquoi ne pas relever le défi et démentir la légende d'Oedipe?

(1) Professeur Dr. Jost Krippendorf fût l'un des tout premiers chercheurs à utiliser la méthode Delphi dans le domaine du tourisme. Son étude, menée en 1977 à la demande du «Fremdenverkehrsdienst des Eidgenössischen Amtes für Verkehr» (Service Tourisme de l'Administra- tiondes Transports Suisse), avait pour objectif la planification prospective du tourisme en Suisse.

(2) Hansruedi Müller, Claude Kaspar, Hanspeter Schmidhauser, Tourismus 2010, Delphi-Umfrage 1991 zur Zukunft des Schweizer Tourismus, Bern/St. Gallen, Forschungsinstitut für Freizeit und Tourismus/Institut für Tourismus und Verkehrswirtschaft, 1991, 64 p.

(3) Forschungsinstitut für Freizeit und Tourismus FIF, Universität Bern, Monbijoustr.29, CH-3011 Bern.

Institut für Tourismus und VerkehrswirtschaftITV, Hochschule St. Gallen Varnbüelstr.19, CH-9000 St. Gallen.

(4) Descriptif de la méthode Delphi: Cf. encart E1.

\section{Les résultats (encart E2)}

- La croissance de la part des vacances d'été en milieu montagnardseraitplus intense que celle des vacances d'hiver en pays chauds.

La part des «sports d'hiver» ne connaîtrait qu'une faible croissance d'ici 2010.

Le pronostic optimiste pour les vacances d'été en montagne est basé sur un aspect écologique (nature préservée en milieu montagnard) et un caractère $*$ sain $*$ de ce type de vacances. L'évolution des prix, l'exploitation de nouvelles destinations et la «surfréquentations des stations touristiques classiques en été avantageraient les vacances d'hiver en pays chauds.

Malgré les changements climatiques, la concurrence des *vacances d'été en hiver» et le cycle de vie des sports d'hiver en général, l'évolution de ce type de vacances serait légèrement positif. Ceci à cause de nouvelles formes de sports d'hiver et de l'aspect «soleil et air frais qui gagnerait de l'importance par rapport à la motivation *neigew.
- Selon les experts, la part du etourisme individuel aisés en Suisse augmenterait d'ici 2010.

LaSuisse serait prédestinée pour cette forme de tourisme à cause de ses entreprises touristiques relativement petites. Qualitéet prixdu tourismeen Suisse s'adresseraient surtout à un segment de touristes à fort pouvoir d'achat.

L'augmentation des parts du tourisme individuel se ferait malgré une plus grande popularité des voyages en groupe et les changements en Europe del'Est qui entraîneraient une hausse du tourisme de groupe.

- D'après les experts, les lieux devacances en Suisse à caractère urbain n'auraient pasà craindre une baisse de la fréquentation à cause d'une future attitude négative envers ces endroits *urbanisés».

Bien qu'une enquête eût souligné une *préférence visuelle» des touristes pour les lieux de vacances à caractère rural, les *villages urbanisés $»$ ne perdraient pas leur attrait. Il semble qu'un paysage et un village jolis, le calme et l'air frais ne suffisent pas à garantir la *joie $»$ des touristes pendant leurs vacances.

- Les experts prévoient un bon avenir pour les séminaires et congrès en Suisse.

Les atouts permettant une évolution favorable seraient une combinaison du travail (congrès) avec des offres de loisir, La Suisse bénéficierait demême des avantages concernant la sécurité, la stabilitế, la propreté et le «savoirfaires.

Les experts moins optimistes mettent le doigt sur les effets de saturation, de concurrence, de l'importance et du perfectionnement croissants des médias électroniques remplaçant les congrès.

Bien que Delphi 1991 prévoit une croissance des congrès en Suisse d'ici 2010 , le rapportévoquela question de savoir si toutes les structures créées récemment dans ce secteur connaîtront un taux d'activité suffisant. 
TABLEAU 1

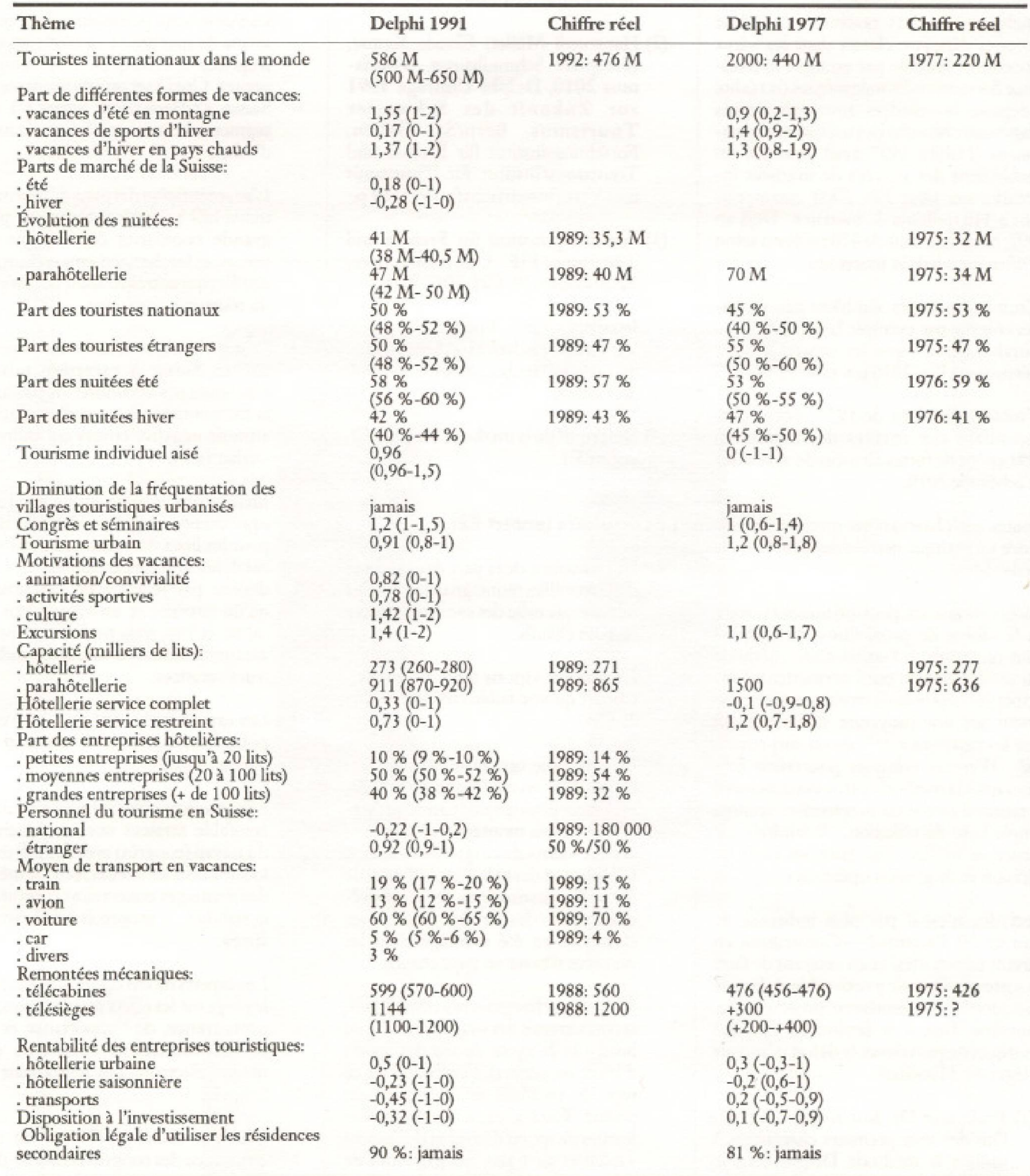

* Le taleau fait ressortir les moyennes arithméthiques et les quartiles (chiffres entre parenthèses).

Plus l'écart entre le quartile inférieur et le quartile supérieur est faible, plus le pronostic semble sûr.

* Les chiffres compris entre -3 et 3 indiquent une baisse ou une croissance du phénomène. ( $-3=$ très forte baisse; 3 très forte croissance). Ce que l'on entend par sfort ou wtrès fort est cependant laissé à l'appréciation de chaque expert. 
- Interrogés sur l'évolution des motivations du tourisme, les experts prévoient une croissance importante de la motivation \&cultures suivie par *animation et convivialités et «activités sportives».

La culture jouerait un rôle plus important entre autres à cause d'une augmentation générale du niveau d'éducation.

L'engouement pour l'animation et la convivialité serait fondé sur un manque actuel d'animation qui implique une participation active, une recherche de la schaleur humaines et un besoin de convivialité des personnes seules et des familles de plus en plus éclatées.

- Les excursions connaîtraient un bel avenir en Suisse.

Une croissance des excursions se justifierait par un besoin accru de «vacances pendant le week-end $\nsim$ et des evacances de courte durée». Un plus grand temps de loisirs entrainerait plus d'excursions, ceci malgré une saturation de l'infrastructure et une nouvelle valorisation du *chez-soiv.

- Quelle sera la part des capacités des petites, moyennes et grandes entreprises de l'hôtellerie Suisse en l'an 2010?

La majorité des experts prévoient une continuation de la tendance qui dure depuis 30 ans: ils annoncent une diminution de la part des petites entreprises hôtelières qui ne constitueraient plus que $10 \%$ en 2010 ( $14 \%$ en 1989). Les moyennes entreprises hôtelières (21 à 100 lits) perdraient également de l'importance (de $54 \%$ en 1989 à $50 \%$ en 2010). Ce sont les grandes entreprises hôtelières de plus de 100 lits qui profiteraient de cette évolution ( $40 \%$ des parts en 2010 par rapport à $32 \%$ en 1989).

Parmi les raisons qu'ont données les experts pour une croissance des grandes entreprises, on citera une meilleure répartition des coûts, la possibilité de présenter une offre élargieetlesmanquesdeconnaissances en management dans les petites et moyennes entreprises hôtelières.
L'affaiblissement de la tendance vers les grandes entreprises pourrait se justifier par une demande croissante pour les entreprises individuelles avec ambiance familiale, par une meilleure flexibilité d'adaptation des petites entreprises, par unmanque de capitaux pour les grandes entreprises.

- La disposition d'investir dans les entreprises touristiques diminuerait légèrement d'ici 2010 selon les experts. Îls donnent tout de même de bonnes chances aux installations de loisir et d'animation (casinos par exemple).

La tendance à la baisse s'expliquerait par de forts besoins en capital par rapport à un faible rendement et à un risque élevé.

- Les expertsdu Delphi 1991 sontd'avis qu'une obligation légale d'utilisation des résidences secondaires (occupation ou location obligatoire) n'existera jamais en Suisse (ou au moins pas avant 2010 ).

Par contre, ils voient une éventuelle obligation d'utilisation des résidences secondaires par la force des choses (augmentation des taxes fiscales ou *taxes de séjour négatives» par exemple).

\section{Forces et faiblesses de la Suisse touristique en l'an 2010 selon Delphi 1991}

\section{Faiblesses}

1- Formation (manque de formation, motivation, qualité) (30)

2- Personnel (recrutement, marché du travail, politique concernant les travailleurs étrangers) (27)

3- Environnement, nature, protection des paysages (20)

4- Hospitalité (16)

5- Problèmes de financementetd'investissement (15)

6- Circulation routière (10)

7- Nature et qualité de l'offre (10)

8- Construction, planification communale et régionale (10)

9- Intervention étatique (9)

10-Marketing, publicité (9)

\section{Forces}

1- Beauté et diversité des paysages (30)

2- Environnement préservé (23)

3- Propreté, exactitude, stabilité proverbiale (22)

4- Systèmes de transport attractifs (17)

5- Bonne qualité et tradition du tourisme (16)

6- Situation géographique (16)

7- Caractère culturel de la Suisse (15)

8- Diversité de l'offre (13)

9- Bonne image, valeur de prestige (6)

10-Bon rapport qualité/prix (3)

* entre parenthèses: le nombre de réponses.

Les problèmes du recrutement et de la formation du personnel se trouvent largement en tête des faiblesses du tourisme suisse selon les experts du Delphi 1991.

Il est cependant étonnant que le rapport qualité/prix ne figure pas dans cette liste des 10 premières faiblesses bien que deux enquêtes parmi lestouristes suisses en 19881989 et 1990-1991 aient *élu» cet aspect «premier problème de l'avenir touristique en Suisses.

En ce qui concerne les forces, les experts et les consommateurs sont d'un avis unanime: la beauté d'un paysage préservé serait le meilleur atout de l'avenir du tourisme en Suisse. Raison de plus pour continuer les efforts de protection de l'environnement. 